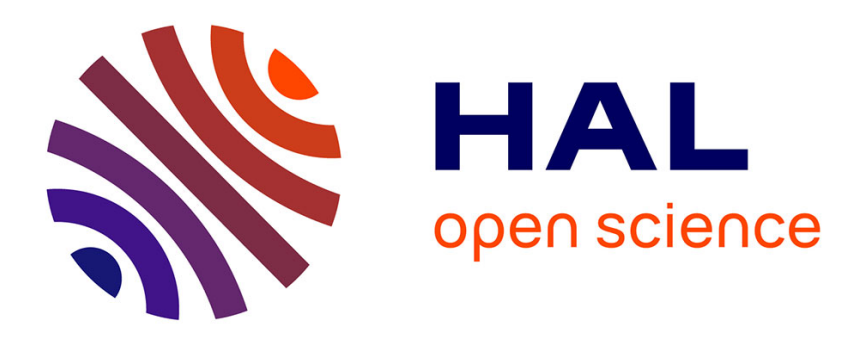

\title{
Contact problems with friction: Models and simulations
}

Frédéric Lebon

\section{To cite this version:}

Frédéric Lebon. Contact problems with friction: Models and simulations. Simulation Modelling Practice and Theory, 2003, 11, pp.449-464. 10.1016/S1569-190X(03)00060-1 . hal-00088317

\section{HAL Id: hal-00088317 https://hal.science/hal-00088317}

Submitted on 12 Feb 2018

HAL is a multi-disciplinary open access archive for the deposit and dissemination of scientific research documents, whether they are published or not. The documents may come from teaching and research institutions in France or abroad, or from public or private research centers.
L'archive ouverte pluridisciplinaire HAL, est destinée au dépôt et à la diffusion de documents scientifiques de niveau recherche, publiés ou non, émanant des établissements d'enseignement et de recherche français ou étrangers, des laboratoires publics ou privés. 


\title{
Contact problems with friction: models and simulations
}

\author{
F. Lebon \\ Laboratoire Mécanique Matériaux Structures, Université Claude Bernard Lyon 1, 82, Bd Niels Bohr, \\ 69622 Villeurbanne Cedex, France
}

\begin{abstract}
This article deals with the modeling and the numerical simulation of contact and friction problems. Some friction formulations are proposed and numerical methods of resolution by finite elements are presented. Two methods are more particularly developed. The first one is based on a formulation using displacements and the second one is based on a mixed formu-lation using displacements and contact forces. Then, some improvements of these two me-thods as well as a comparison of their respective performances are presented.
\end{abstract}

Keywords: Unilateral contact; Friction; Variational formulations; Numerical methods

\section{Introduction}

Friction is a natural occurrence that affects almost all objects in motion. The miracle of fire by friction was the first manifestation of friction for our ancestors, ten thousand years ago. In the Egyptian antiquity, people used grease in order to move stones on inclined planes. Since then with active friction compensation for motors and friction welding machines, extensive research has attempted to develop models that accurately predict the friction behavior. We use the expression "friction force" to speak about the resisting force tangential to the interface between two bodies when, under the action of an external force, one body moves or tends to move to the other (two pieces of wood for the Aboriginal people of Australia, stone and ground for Egyptians). From an industrial and technological point of view, one

E-mail address: lebon@iutal2m.univ-lyon1.fr (F. Lebon). 
can fight against friction or take advantage of friction. On one hand, our ancestors have used the friction in order to obtain fire. In a friction welding machine, friction is used to generate heat and to produce a high integrity joint between similar or dissimilar metals. The advantages are both metallurgical and physical. On the other hand, Egyptians wanted to shift heavy stones, and nowadays, technologies have been developed in order to moderate friction effects. For example, friction composites, which use auto-lubrication, have been recently developed. Other techniques allow to obtain a coating able to restore the initial geometry before friction effects on the contact surface.

This paper outlines modeling and computational methods for studying the contact between deformable solids. Indeed, many structural analysis problems are concerned with frictional contact phenomena, specifically for problems concerned with structure assembly. These problems are difficult to formulate and even more to solve because they are characterized by non-linear relations between displacements or rates of displacements and forces on a part of the boundary, and usually governed by multi-valued tribological laws. Some numerical solutions can lead to problems with non-linear constraints or non-symmetric operators. These drawbacks become crucial for very large problems involving three dimensional discretization or/and time evolution.

In the first part of this paper, the contact is characterized by the Signorini's law and the friction by the semi-empirical Coulomb's law, which is convenient for a large class of solids and structures.

In order to solve the problem, two families of algorithms are presented.

The first one is based on a fixed point algorithm on the implicit friction term [51]. At each iteration of the fixed point algorithm, we have to solve an optimization problem with constraints. The problem being not differentiable is classically solved by relaxation procedures. This algorithm has been used by the author and his collaborators for the analysis of a complex assembly in reactor technology [36] and for the analysis of coupling sleeves in shape memory alloys [47]. The numerical results have been compared with experimental data and a validation of the model has been obtained for the first application. This method is very simple to be implemented and to be coupled with complex behaviors. It is a robust method and the convergence does not depend much on the friction coefficient. Improvements of the algorithm have been done using multi-grid methods [33]. These methods are known to be efficient and robust. To put it more precisely, a super-convergence is obtained.

The second algorithm is based on a mixed formulation and leads to non-linear and non-differentiable problems. These problems are solved by a generalized Newton method (GNM). The tangent matrix of the system is non-symmetric, non-positive definite, ill-conditioned and with zeros on the diagonal. Consequently, it is necessary to design appropriate preconditioners [5]. The improvement of this algorithm needs the use of parallel computers and, therefore, the implementation of domain decomposition methods. Industrial problems such a system of rolling shutters and a leaf spring-dashpot suspension system have been analyzed in former papers [1].

The last part of the paper is devoted to a comparison between the methods presented above. The academic example of a composite long bar in contact with a rigid 
obstacle is treated. This problem has the advantage to present complex contact zones and very ill-conditioned stiffness matrices. An analysis of efficiency and robustness of the two algorithms is given. Note that the two algorithms have been implemented in the finite element software Modulef [11] and computations have been done on various types of platforms (IBM SP2, Sun station, Windows PC).

\section{Formulations}

\subsection{Friction modeling}

One can consider that the first attempt of interfaces modeling is due to Amontons [8] in 1699, analysis resumed by Coulomb [17] in 1785. In these works, the real interface is untidy and only the surfaces in contact are considered. This law called Coulomb's law, saying that a sliding appears when the module of the tangential force is proportional to the normal force of contact, even nowadays is subjected to intense researches for the theoretical level and for the numerical level $[18,20,29,49,58]$. It is generally taken into account in a small satisfactory way in industrial computer codes. Note (see [30,42], and references therein) that nowadays different types of simulation methods are used (finite element methods, boundary element method,...) and numerous types of problems are treated (rolling contact, impact and dynamics, instabilities, oscillations, waves,...). Furthermore, during the last decades, the law of Coulomb knew attempts of improvement and modification (let us quote the laws of compliance [44] or the laws of adhesion [54]).

Another approach, much more recent, consists in trying to precisely take into account the microstructures of the interfaces, that one generally qualifies as laws of the third body. In the same order of idea, a glue between two substrata can also be considered as a third body having mechanical and physical characteristics very different from both others. It is the same for the interfaces between the various constituents of a composite. The problem seems to be very difficult; for example, the question of the real dimensions of the interface as well as the determination of its mechanical characteristics are complex problems. Another difficulty appears when these interfaces are taken into account in a design by finite elements. The size of the interface being generally very weak according to the structures dimensions, numerically, two fundamental problems in scientific computation appear: the cost and the precision of the computations. The cost becomes very high not only due to the non-linearities of the problem but also due to a very high number of degrees of freedom, due to the small size of the interface. This aspect becomes major for problems of structures assembly for which the interfaces are very numerous. From the economical point of view, the costs can seem too important. The other aspect, which concerns the precision, can turn out catastrophic in a strictly mechanical point of view and can question the design. To the size of the problems, it adds the very strong variations of the physical characteristics of materials. These variations pull what numericians call a bad conditioning of the calculations. Indeed, in practice, weak variations of the data, that are difficult to obtain experimentally, can lead to very strong variations of the results. 
In a theoretical way, one can show that these variations are strongly stressed by the size of the calculations. Such or such characteristic, such or such criterion of ruin or collapse can be perfectly under or overestimated in a considerable way after long days (month) of calculation.

At the end of the 1980s, another strategy has appeared [55]. This strategy consists in noticing that the size of the interface is small and that one can consider it as "a small parameter". From the mathematical point of view the framework is perfectly identified. One proceeds that one calls an asymptotic study $[9,19,52]$; the small parameter (the thickness), eventually the small parameters, aim towards zero, and the limit problem is studied. In a general way, the interface disappears from a geometrical point of view but is kept in the form of a mechanical constraint (we will call it law of interface) through a surface energy. In this case, a condition between the two bodies appears on this surface. Usually, this condition connects the stress vector $\sigma n$ to the jump of displacements $[u]$, where $\sigma$ is the stress tensor. Numerous studies have been devoted to this subject $[21,24,31,34,35,37-39,45,46,55,59,60]$.

\subsection{Generalities and notations}

In the following parts of the paper, we use the classical terminology; a problem is qualified as primal if it involves displacements, dual if it involves forces and mixed if it involves both displacements and forces. The term "condensed" will be added to precise that the unknowns (displacements or forces) are researched on the contact boundary. Considering the classical duality schema presented in Fig. 2 [27,28], we recall that two different duality products are defined, in the volume and on the boundary. Two operators from the volume to the boundary and from the boundary to the volume are necessary. The particularity of a contact problem is to mix variables on the boundary and variables in the domain.

In this section Coulomb's and Signorini's laws are precised. We work in quasistatic and in two dimensions. Let us consider a deformable body and $\Omega$ the interior of this solid where body forces $F$ are applied. In order to simplify, the solid is supposed to be elastic and in contact with a rigid plane on a part of the boundary $\Gamma_{\mathrm{c}}$ (Fig. 1). For this formulation, $\Gamma_{\mathrm{c}}$ is defined as the initial real contact area and only a loss of contact on $\Gamma_{\mathrm{c}}$ will be allowed. Nevertheless, generalizations are possible: contact between deformable bodies, large sliding,...

Let us consider $H^{1}(\Omega)$ the Sobolev space of order $1, H$ and $H_{0}$ the subspaces of $\left(H^{1}(\Omega)\right)^{2}$ defined by

$$
\begin{aligned}
& H=\left\{v \in\left(H^{1}(\Omega)\right)^{2}, \quad \gamma v=U \quad \text { on } \Gamma_{\mathrm{d}}\right\}, \\
& H_{0}=\left\{v \in\left(H^{1}(\Omega)\right)^{2}, \quad \gamma v=0 \quad \text { on } \Gamma_{\mathrm{d}}\right\},
\end{aligned}
$$

$\gamma$ is the trace operator on the boundary $\Gamma$ and $\Gamma_{\mathrm{d}}$ is the part of the boundary where a displacement $U$ is prescribed. Forces $f$ are applied on the part of the boundary $\Gamma_{\mathrm{f}}$.

On the part of the boundary, $\Gamma_{\mathrm{c}}$ a local referential is introduced $(n, t)$ where $n$ is the external unit vector to $\Omega$. In this referential, the trace of displacement $v$ and the stress vector $\sigma n$ are decomposed. Let us denote $v_{\mathrm{N}}$ the normal component of the dis- 


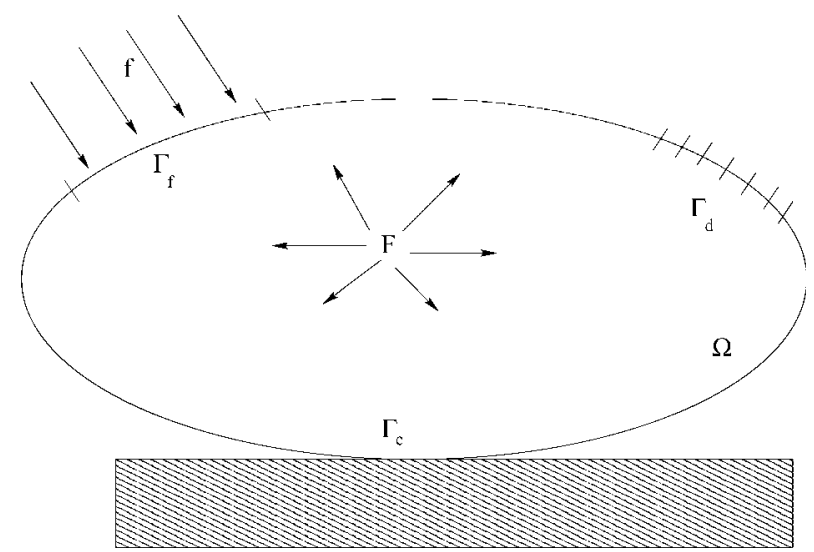

Fig. 1. The famous patatoïd in contact.

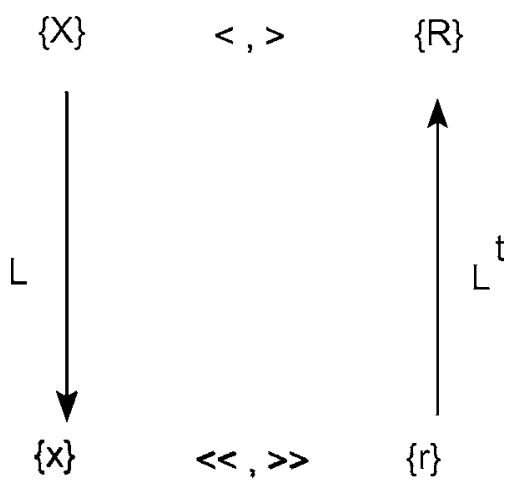

Fig. 2. Schema of duality: $X$ space of displacements, $x$ space of contact displacements, $R$ space of stresses, $r$ space of contact forces.

placement, $v_{\mathrm{T}}$ its tangential part and $\sigma_{\mathrm{N}}, \sigma_{\mathrm{T}}$ respectively the normal and the tangential component of the stress vector. We define $\mathscr{K}$ the convex of contact of kinematically admissible fields $\mathscr{K}=\left\{v \in H, v_{\mathrm{N}} \leqslant 0\right.$ on $\left.\Gamma_{\mathrm{c}}\right\}$. We introduce the generalized-standard-materials formalism [25]. The index $\mathrm{r}$ (resp. ir) indicates reversible part (resp. irreversible) of the stress. The volume free energy, non-differentiable (i.e. non-smooth), taking into account unilateral contact is written

$$
W(e)=\frac{1}{2} e A e+I_{\mathscr{K}}(v),
$$

where $I_{\mathscr{K}}$ is the indicatrix function of convex $\mathscr{K}$.

Unilateral contact conditions (Signorini's conditions) are taken into account adding this indicatrix function to the elastic energy. Using usual notations $e$ is the strain tensor and $A$ the fourth-order elasticity tensor. The pseudo-potential of dissipation associated to Coulomb's law is written 


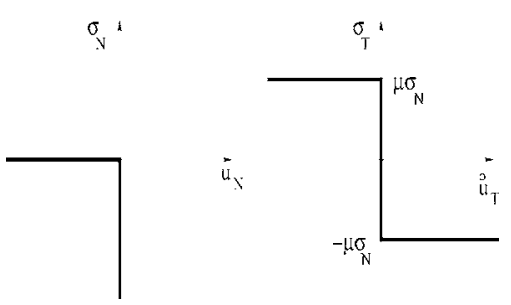

Fig. 3. Contact laws of Signorini and Coulomb.

$$
D\left(\dot{v}_{\mathrm{T}}\right)=\mu\left|\sigma_{\mathrm{N}}^{\mathrm{r}}\right|\left\|\dot{v}_{\mathrm{T}}\right\|
$$

where $\dot{v}$ is the time derivative of displacement field $v$.

State laws and complementarity laws are written $(\partial$ is the sub-differential operator $[14,43])$

$$
\begin{aligned}
& \sigma=\sigma^{\mathrm{r}}=A e(v) \quad \text { in } \Omega, \\
& \sigma_{\mathrm{N}}=\sigma_{\mathrm{N}}^{\mathrm{r}} \in \partial I_{\mathscr{K}}\left(u_{\mathrm{N}}\right) \quad \text { on } \Gamma_{\mathrm{c}}, \\
& \sigma_{\mathrm{T}}=\sigma_{\mathrm{T}}^{\mathrm{ir}} \in \partial D\left(\dot{u}_{\mathrm{T}}\right) \quad \text { on } \Gamma_{\mathrm{c}} .
\end{aligned}
$$

Eq. (4) gives the complementarity problem of Signorini and the Coulomb's law (see Fig. 3). Let us denote $u_{0}$ the initial displacements fields. Equilibrium equations, Signorini's and Coulomb's laws and behavior law lead to the variational formulation (coupled variational inequalities [16])

$$
\begin{aligned}
& u_{0} \in H \quad \text { be given, find } u \in V=H^{1}(0, T ; \mathscr{K}) \quad \text { such that } u(0)=u_{0} \quad \text { in } \Omega \\
& a(u, v-\dot{u})+j(u, v)-j(u, \dot{u}) \leqslant l(v-\dot{u}), \quad \forall v \in H_{0} \\
& \int_{\Gamma_{\mathrm{c}}} \sigma_{\mathrm{N}}(u)\left(z_{\mathrm{N}}-u_{\mathrm{N}}\right) \mathrm{d} l \leqslant 0, \quad \forall z \in \mathscr{K}
\end{aligned}
$$

with $j(u, v)=-\int_{\Gamma_{\mathrm{c}}} \mu \sigma_{\mathrm{N}}(u)\left|\dot{v}_{\mathrm{T}}\right| \mathrm{d} l$,

$$
l(v)=\int_{\Omega} F v \mathrm{~d} x+\int_{\Gamma_{\mathrm{f}}} f \gamma v \mathrm{~d} l
$$

and $a(u, v)=\int_{\Omega} e(u) A e(v) \mathrm{d} x$.

Using a finite difference,

$$
\dot{u}\left(t^{k+1}\right) \simeq \frac{u^{k+1}-u^{k}}{\Delta t}=\frac{\Delta u^{k}}{\Delta t},
$$

with $u^{k}=u\left(t_{k}\right)$, we have at time $t_{k+1}$.

Find $u^{k+1}=u^{k}+\Delta u^{k} \in \mathscr{K}$ such that

$$
a\left(u^{k+1}, v-\Delta u^{k}\right)+j\left(u^{k+1}, v\right)-j\left(u^{k+1}, \Delta u^{k}\right) \leqslant l\left(v-\Delta u^{k}\right), \quad \forall v \in \mathscr{K} .
$$




\subsection{Fixed point method FPM}

In the following sections, indices $k$ (time) are omitted (static formulation). The former problem is expressed as a fixed point problem on the sliding limit $[15,22]$

$$
\lambda \rightarrow-\mu \sigma_{\mathrm{N}}(u(\lambda))
$$

$u$ is the solution of a variational inequation similar to the former one, where $j(\cdot, \cdot)$ is replaced by $j(\cdot)$ defined by

$$
j(v)=\int_{\Gamma_{\mathrm{c}}} \lambda\left|v_{\mathrm{T}}\right| \mathrm{d} l .
$$

The last problem is equivalent to the minimization in $\mathscr{K}$ of $L(v)=\frac{1}{2} a(v, v)-$ $l(v)+j(v)$. It is possible to regularize the non-differentiable term in the minimization problem, the absolute value is replaced by a smooth function. For example, $\sqrt{x^{2}+\epsilon^{2}}$ or $\epsilon \operatorname{Ln}(\operatorname{ch}(x / \epsilon))$ can be chosen [33,50]. This regularization does not modify numerical process structures. Usually, the problem is discretized by a linear interpolation (Finite Element Method). Let $h$ the step of discretization. The displacement leads to a vector of dimension $N_{h}$. If $I_{h}$ is the set of degrees of freedom concerned by friction, the fixed point iteration consists in finding the vector of dimension $I_{h}, \lambda_{h}$, fixed point of the discretized previous problem. The algorithm (which is convergent [40]) is written:

- Step 0 Initialization

$$
u^{0} \text { be given }
$$

- Step 1 Fixed Point (Iteration k)

$$
\lambda^{k} \rightarrow-\mu \sigma_{\mathrm{N}}\left(u\left(\lambda^{k-1}\right)\right)
$$

- Step 2 Minimization (Relaxation)

○ Step A Initialization $u^{k, 0}=u^{k}$

- Step B Resolution (iteration $\mathrm{n},\left(a_{i j}\right)$ is the stiffness matrix)

For a normal component of a contact node

$$
\begin{aligned}
& \left.u_{i}^{k, n+\frac{1}{2}}=\frac{1}{a_{i i}} f_{i}-\sum_{j=1}^{j=i-1} a_{i j} u_{j}^{k, n}-\sum_{j=i+1}^{j=N_{h}} a_{i j} u_{j}^{k, n+1}\right) . \\
& \text { If } u_{i}^{k, n+\frac{1}{2}} \geqslant 0 \quad \text { then } u_{i}^{k, n+1}=0 .
\end{aligned}
$$

For a tangential component of a contact node

$$
\begin{aligned}
& u_{i}^{k, n+1}=\frac{1}{a_{i i}}\left(f_{i}-\sum_{j=1}^{j=i-1} a_{i j} u_{j}^{k, n}-\sum_{j=i+1}^{j=N_{h}} a_{i j} u_{j}^{k, n+1}+\lambda_{i}^{k} \epsilon\left(u_{i}^{k, n+1}\right)\right) . \\
& \text { If } x \geqslant 0, \epsilon(x)=1, \quad \text { if } x \leqslant 0, \epsilon(x)=-1, \quad \text { if } x=0, \epsilon(x)=0 .
\end{aligned}
$$

For a component of a free node (not concerned by the contact)

$$
\left.u_{i}^{k, n+1}=\frac{1}{a_{i i}} f_{i}-\sum_{j=1}^{j=i-1} a_{i j} u_{j}^{k, n}-\sum_{j=i+1}^{j=N_{h}} a_{i j} u_{j}^{k, n+1}\right) \text {. }
$$


- Step C Convergence test

Yes: $u^{k+1}=u^{k, n+1}$, Go to Step 3

No: Go to Step B

- Step 3 Convergence Test

Yes $=$ End; No $=$ Go to Step 1.

At each iteration $k$, we have to minimize the energy in the discretized convex $\mathscr{K}_{h}$. This step is done using a relaxation procedure written above. The functional being strictly convex, the minimization process has an unique solution.

\subsection{FPM: improvements and applications}

The first improvement for the fixed point algorithm is the use of a diagonal procedure i.e. the internal optimization problem is only partially solved. Another improvement is to introduce a relaxation parameter or an Aitken procedure [50]. A third improvement is the use of a condensation method (or Schür complement). In this case, the problem is condensed on the interface. This technique is the foundation of a class of very efficient parallel algorithms [36,51]. The last improvement that we have tested is the use of multigrid techniques [33]. These algorithms have been used for the analysis of a complex assembly in reactor technology [36], for the analysis of coupling sleeves in shape memory alloys [47] and for the study of piezoelectric sensors [13]. In the first application, the numerical results has been compared with experimental data and a validation of the Coulomb's law has been obtained. The two other applications have shown the simplicity of coupling this method to complex behaviors.

\subsection{Generalized Newton method}

The discretized problem is written as an equilibrium equation, introducing the contact forces $\lambda$, find $u$ and $\lambda$ such that

$$
\begin{aligned}
& -A u+f+\mathscr{F}(u, \lambda)=0, \\
& -\frac{1}{r}(\lambda-\mathscr{F}(u, \lambda))=0,
\end{aligned}
$$

where $\mathscr{F}(u, \lambda)$ is the friction map. In the case of a node in contact with a rigid obstacle, we have $[3,5]$

$$
\mathscr{F}(u, \lambda)=\operatorname{proj}_{R_{-}}\left(\tau_{\mathrm{N}}\right) n+\operatorname{proj}_{C\left(\operatorname{proj}_{R_{-}}\left(\tau_{\mathrm{N}}\right)\right)}\left(\tau_{\mathrm{T}}\right),
$$

proj is the projection operator, $C\left(\lambda_{\mathrm{N}}\right)$ the Coulomb's cone and $r$ is a parameter. $\tau_{\mathrm{N}}$ et $\tau_{\mathrm{T}}$ are given by

$$
\tau_{\mathrm{N}}=\lambda_{\mathrm{N}}+r u_{\mathrm{N}}, \quad \tau_{\mathrm{T}}=\lambda_{\mathrm{T}}+r u_{\mathrm{T}} .
$$

The non-linear problem is thus written

$$
\mathscr{D}(x)+\mathscr{U}(x)=0,
$$


with

$$
\mathscr{D}(x)=\left[\begin{array}{c}
-A u+f \\
-\frac{1}{r} \lambda
\end{array}\right] \text { and } \mathscr{U}(x)=\left[\begin{array}{c}
\mathscr{F}(u, \lambda) \\
\frac{1}{r} \mathscr{F}(u, \lambda)
\end{array}\right] .
$$

This system is solved by a GNM

$$
x^{i+1}=x^{i}-\left(\nabla \mathscr{D}\left(x^{i}\right)+\partial \mathscr{U}\left(x^{i}\right)\right)^{-1}\left(\mathscr{D}\left(x^{i}\right)+\mathscr{U}\left(x^{i}\right)\right)
$$

$\partial \mathscr{U}\left(x^{i}\right)$ is a point of the Jacobian matrices set. The linearized system (18) is solved by a conjugate squared method [53]. The algorithm is summed up by

- Step 0 Initialization

$$
x^{0}=\left(u^{0}, \lambda^{0}\right) \text { be given }
$$

- Step 1 Resolution (Non-symmetric linear system)

$$
y^{i+1}=\left(\nabla \mathscr{D}\left(x^{i}\right)+\partial \mathscr{U}\left(x^{i}\right)\right)^{-1}\left(\mathscr{D}\left(x^{i}\right)+\mathscr{U}\left(x^{i}\right)\right)
$$

- Step 2 Actualization

$$
x^{i+1}=x^{i}-y^{i+1}
$$

- Step 3 Convergence Test

Yes $=$ End; No $=$ Go to Step 1 .

\subsection{GNM: improvements and applications}

The first improvement of the GNM is the use of preconditioners as incomplete factorization $[4,5]$. Coarse/fine techniques, similar to multigrid methods, have been developed [5,23]. More recently, an element-by-element procedure has been proposed. This technique consists in a factorization of the tangent matrix from the factorization of the elementary matrices. The advantage of this method is to be strongly parallel $[2,10,26]$. At least, domain decomposition methods have been implemented in order to treat the problem with a large number of degrees of freedom [1]. Using these algorithms, we have analyzed industrial problems such as a system of rolling shutters and a leaf spring-dashpot suspension system. In the first case, the body is a succession of slats. We have shown that friction and gaps between the slats have a crucial importance on the structure behavior. The second application is a pile of four beams in contact. These beams are linked by deformable bolts. We have shown that we need to take into account dynamics effects to obtain accurate contact distribution and tensions in the bolts. Note that existence results have been studied using this formulation [7].

\subsection{Remarks on dual formulations}

A third approach is possible by using a dual formulation, in which the unknowns are the tensions (the contact forces for the condensed version). The dual formulation of the problem has been introduced by Haslinger and Panagiotopoulos in 1984 [48] and Telega in 1988 [56,57]. This formulation is used by a poor number of authors from a numerical point of view $[12,32,41]$. 


\section{Comparisons}

\subsection{The benchmark: a stratified in contact with a rigid obstacle}

This section is devoted to the comparison of the two methods presented above, the first comparison in the literature. The problem treated in this section is a stratified composite in contact with a rigid plan [6]. This stratified is made by a succession of hard and soft folds. The Young's modulus of the hard component is a hundred times larger than the coefficient of the soft one. The layers are orthogonally oriented to the contact area (Fig. 4). The Poisson's ratio is equal to 0.3 . For symmetry reasons, only a half-bar is considered. This example, which is a variant of a classical benchmark [51], is treated in plane stresses. Two kinds of elementary patterns are considered: a succession of soft and hard folds (pattern 1) and a succession of hard and soft folds (pattern 2). These two patterns give various results, both for numerical and mechanical points of view. In our numerical applications, we consider 32 elementary patterns and 16962 degrees of freedom (64 contact nodes). This example, which is very ill-conditioned, allows to compare the efficiency of the two algorithms FPM and GNM.

\subsection{Operation numbers}

In order to compare the two methods, we need to observe that the cost of each method depends on the number of global iterations and on the cost of internal solvers (Relaxation for FPM and Conjugate Gradient for GNM). In this paragraph, we focus on the number of operations for the two internal solvers.

Classically, one iteration of the relaxation procedure corresponds to one product between a sparse matrix and a vector. If $N_{h}$ is the number of nodes and $c_{h}$ the ave-

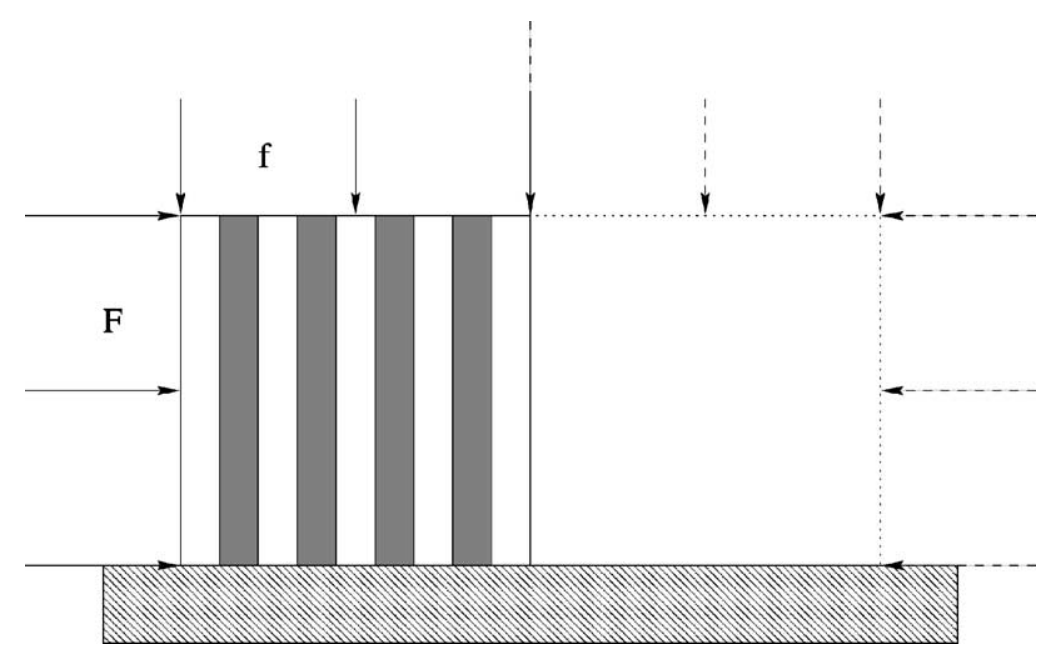

Fig. 4. Compression of a composite on a rigid body. 
raged value of the coefficients not equal to zero for each line of the matrix, the number of operation $K_{\mathrm{REL}}$ verifies

$$
K_{\mathrm{REL}} \approx 4 c_{h} N_{h} .
$$

Generalized conjugate gradient methods corresponds to two products between a sparse matrix and a vector. Due to the mixed formulation the number of nodes is equal to $N_{h}+n_{h}$, where $n_{h}$ is the number of contact nodes. Thus, the number of operation $K_{\mathrm{GCG}}$ for this method is given by

$$
K_{\mathrm{GCG}} \approx 8 c_{h}\left(N_{h}+n_{h}\right) .
$$

For a low number of contact nodes, the cost of the generalized conjugate gradient is twice the one of the relaxation method.

\subsection{External iterations comparisons: FPM versus $G N M$}

On Fig. 5, we can see the evolution of the global iterations number for the two methods in relation to the coefficient of friction. One can observe that, usually, the GNM is more efficient than the FPM and that the two methods have the same behavior for the two kinds of patterns. We observe a difference between $20 \%$ and $60 \%$ in the number of iterations of the two external solvers for standard values of the friction coefficient. Nevertheless, for high level of friction coefficient, the FPM is more robust than the other method. For large friction coefficients the convergence of the GNM is not ensured. To conclude, the two methods are complementary. For 'small' values of friction coefficient $(\leqslant 1)$, the GNM will be used and for larger values of friction coefficient $(\geqslant 1)$, the FPM will be preferred.

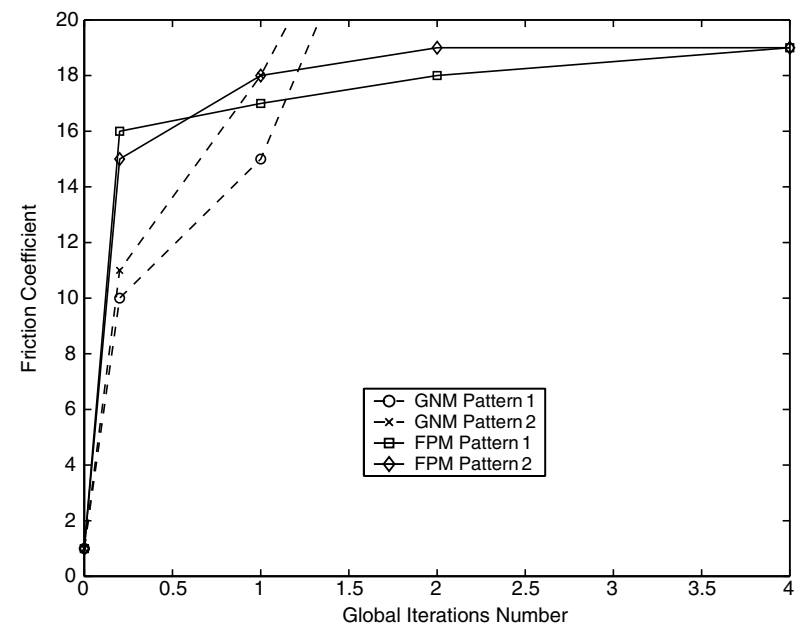

Fig. 5. Fixed point and Newton iterations versus $\mu$. 


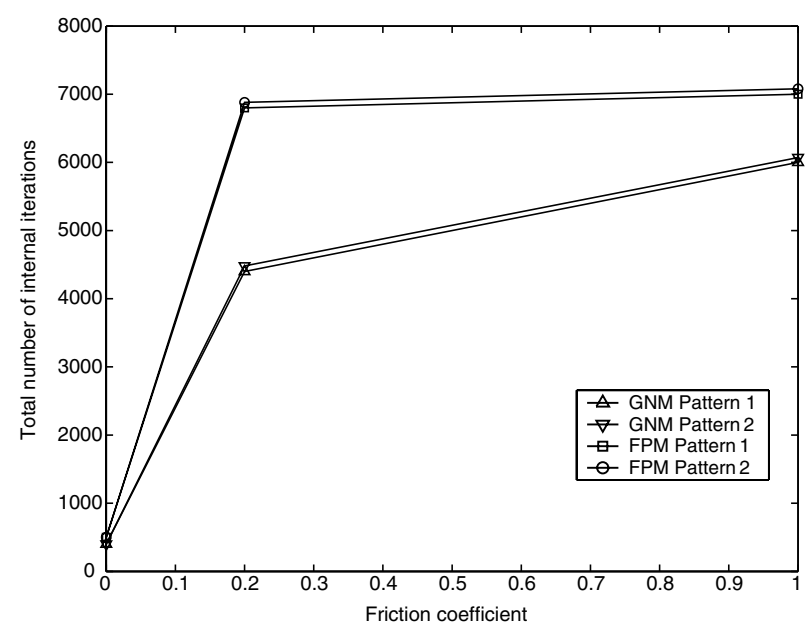

Fig. 6. Relaxation iterations and gradient "normalized" iterations versus $\mu$.

\subsection{Internal iterations comparisons: relaxation versus conjugate gradient}

Fig. 6 shows the internal iteration number for values of the friction coefficient lower than 1. We observe that the internal solver associated to the GNM (here the conjugate gradient squared method) is more efficient than the internal solver associated to the FPM (relaxation procedure). Note that in this figure the number of conjugate gradient iterations is normalized (multiplied by $K_{\mathrm{GCG}} / K_{\mathrm{REL}}$ ) to take into account the various numbers of operations of each method. On one hand, taking into account the fact that the conjugate gradient squared method is twice expensive than the relaxation procedure, between $10 \%$ and $35 \%$ of difference is obtained between the two internal solvers in terms of operations. This difference decreases when the friction coefficient increases. On the other hand, if we compare these internal solvers, we can see that relaxation procedures seem more robust than conjugate gradient methods i.e. the total number of iterations does not strongly depend on the friction coefficient. In conclusion, we can add that these methods need acceleration procedures in order to converge. Classically, pre-conditioners associated to gradient methods are more expensive than over-relaxation associated to relaxation procedure.

\section{Conclusions and perspectives}

In this paper, different models for interfaces have been presented and various formulations of the Signorini-Coulomb's problem have been introduced. Two algorithms (FPM and GNM) have been described in order to numerically solve the problem and a first comparison between these two methods dedicated to solve frictional contact problems has been done. 
We have shown, that for a large class of friction coefficients, the GNM associated to a mixed formulation is more efficient than the FPM associated to a primal formulation. Nevertheless, for large values of friction coefficient the second method seems more robust than the first one and will be preferred in this case. The computations have been done on various platforms. The portability of these methods is very great and the previous remarks on the respective behaviors of the two algorithms are valid independently of the platform.

Future extensions to this work are of two kinds, numerical and theoretical. The treatment of large sliding, the treatment of problems with a very large number of contacts as granular materials and the implementation of other non-linear contact laws could be studied. Another work will be a comparison between the two methods presented above and the D-PANA algorithm associated to the dual formulation [12].

\section{Acknowledgements}

I make a point of thanking Professor Franco Maceri for his invitation in his department during the year 2000-2001. I would like to thank Professor Paolo Bisegna for the numerous and profitable discussions on contact and friction. Most of this work was carried out in Montpellier. I particularly thank Professor Olivier Maisonneuve for all these years in his laboratory as well as researchers who collaborated with me.

This work was developed within the framework of Lagrange Laboratory, an European Research Group between CNRS, CNR, University of Rome "Tor Vergata", University of Montpellier II, ENPC and LCPC.

\section{References}

[1] K. Ach, P. Alart, M. Barboteu, F. Lebon, B. Mbodji, Parallel frictional contact algorithms and industrial applications, Comp. Meth. Appl. Mech. Eng. 177 (1999) 169-181.

[2] P. Alart, M. Barboteu, F. Lebon, Solution of frictional contact problems by an EBE preconditioner, Comput. Mech. 20 (1997) 370-378.

[3] P. Alart, A. Curnier, A mixed formulation for frictional contact problems prone to Newton like solution methods, Comp. Meth. Appl. Mech. Eng. 92 (1991) 353-375.

[4] P. Alart, F. Lebon, Multigrid method applied to mixed formulation for frictional contact problem, in: M.H. Aliabadi, C.A. Brebbia (Eds.), Contact Mechanics, Computational Techniques, Computational Mechanics Publications, 1993, pp. 227-234.

[5] P. Alart, F. Lebon, Solution of frictional contact problems using ILU and coarse/fine preconditioners, Comput. Mech. 16 (1995) 98-105.

[6] P. Alart, F. Lebon, Numerical study of a stratified composite coupling homogenization and frictional contact, Math. Comp. Model. 28 (1998) 273-286.

[7] P. Alart, F. Lebon, F. Quittau, K. Rey, Frictional contact problem in elastostatics: revisiting the uniqueness condition, in: M. Raous, M. Jean, J.J. Moreau (Eds.), Contact Mechanics International Symposium, Plenum Press, 1994, pp. 19-23.

[8] G. Amontons, Sur l'origine de la résistance dans les machines, Mém. l'Acad. Roy. (1699) 206-222.

[9] H. Attouch, Variational Convergence for Functions and Operators, Pitman Advanced Publishing Program, 1984. 
[10] M. Barboteu, P. Alart, F. Lebon, An improved EBE preconditioner for elastostatics, J. Appl. Mech. 65 (1998) 531-533.

[11] M. Bernadou et al., Modulef une Bibliothèque Modulaire D'éléments Finis, Inria, 1988.

[12] P. Bisegna, F. Lebon, F. Maceri, D-PANA: a convergent block-relaxation solution method for the discretized dual formulation of the Signorini-Coulomb contact problem, CR Acad. Sci. Sér. I, Paris 333 (2001) 1053-1058.

[13] P. Bisegna, F. Lebon, F. Maceri, The unilateral frictional contact of a piezoelectric body with a rigid support, in: J.A.C. Martins, M.M. Marques (Eds.), Contact Mechanics International Symposium, Kluwer, 2002.

[14] F.H. Clarke, Optimization and Non-Smooth Analysis, first ed., Wiley, 1982.

[15] M. Cocu, Existence of solutions of Signorini problems with friction, Int. J. Eng. Sci. 22 (1984) 567-575.

[16] M. Cocu, E. Pratt, M. Raous, Formulation and approximation of quasistatic frictional contact, CR Acad. Sci. Sér. I, Paris 320 (1995) 1413-1420.

[17] C.A. Coulomb, Théorie de machines simples, Mém. Math. Phys. l'Acad. Roy. 10 (1785) 161-342.

[18] A. Curnier (Ed.), Contact Problems, Presses Universitaires Romandes, 1992.

[19] G. Dal Maso, An Introduction to $\Gamma$-Convergence, Birkhauser, 1993.

[20] G. Del Piero, F. Maceri (Eds.), Unilateral Problems in Structural Analysis IV, Birkhauser, Verlag, 1991.

[21] S. Dumont, F. Lebon, A. Ould-Khaoua, A numerical tool for periodic heterogeneous media: application to interface in Al/SiC composites, J. Appl. Mech. 67 (2000) 214-217.

[22] G. Duvaut, J.L. Lions, Inequalities in Mechanics and Physics, Springer-Verlag, 1976.

[23] C. Farhat, N. Sobh, A coarse/fine preconditioner for very ill-conditioned finite element problems, Int. J. Numer. Meth. Eng. 28 (1989) 1715-1723.

[24] G. Geymonat, F. Krasucki, Analyse asymptotique du comportement en flexion de deux plaques collées, CR Acad. Sci. Sér. I, Paris 325 (1997) 307-314.

[25] B. Halphen, Q.S. Nguyen, Sur les matériaux standards généralisés, J. Theor. Appl. Mech. 14 (1975) 39-63.

[26] J.T.R. Hughes, I. Levit, J.M. Winget, An element-by-element solution algorithm for problems of structural and solid mechanics, Comp. Meth. Appl. Mech. Eng. 36 (1983) 241-254.

[27] M. Jean, Un cadre abstrait pour l'espace vectoriel topologique ordonné $W^{1, p}(\Omega)$ et quelques uns de ses bornés, Travaux du séminaire d'analyse convexe, exposé 7, 1975.

[28] M. Jean, Some complete lattices of $W^{1, p}(\Omega)$, Boll. Dell. Unione Mat. Ital. 5 (1976) 186-195.

[29] K.L. Johnson, The application of the shakedown principles in rolling and sliding contact, Eur. J. Mech. A Solids 11 (1992) 155-172.

[30] J.J. Kalker (Ed.), Three-Dimensional Elastic Bodies in Rolling Contact, Kluwer Academic Press, 1990.

[31] A. Klarbring, Derivation of the adhesively bonded joints by the asymptotic expansion method, Int. J. Eng. Sci. 29 (1991) 493-512.

[32] A. Klarbring, A. Mikelic, M. Shillor, On friction problems with normal compliance, Non-linear Anal. Theor. Meth. Appl. 13 (1989) 935-955.

[33] F. Lebon, Two-grid method for regularized frictional elastostatics problems, Eng. Comput. 12 (1995) 657-664.

[34] F. Lebon, C. Licht, F. Zaittouni, Asymptotic modelling of interface taking into account contact conditions, Part 1: Asymptotic expansions and numerical implementation, Int. J. Solids Struct., submitted.

[35] F. Lebon, A. Ould-Khaoua, C. Licht, Numerical study of soft adhesively bonded joints in finite elasticity, Comput. Mech. 21 (1998) 134-140.

[36] F. Lebon, M. Raous, Friction modelling of a bolted junction under internal pressure loading, Comp. Struct. 43 (1992) 925-933.

[37] C. Licht, Comportement asymptotique d'une bande dissipative mince de faible rigidité, CR Acad. Sci. Sér. I, Paris 317 (1992) 429-433.

[38] C. Licht, G. Michaille, Une modélisaton du comportement d'un joint collé élastique, CR Acad. Sci. Sér. I, Paris 322 (1996) 295-300. 
[39] C. Licht, G. Michaille, A modeling of elastic adhesive bonded joints, Adv. Math. Sci. Appl. 7 (1997) 711-740.

[40] C. Licht, E. Pratt, M. Raous, Remarks on a numerical method for unilateral contact including friction, in: Unilateral Problems in Structural Analysis, International Series on Numerical Mathematics, 1991, pp. 129-144.

[41] F. Maceri, P Bisegna, The unilateral frictionless contact of a piezoelectric body with a rigid support, Math. Comp. Model. 28 (1998) 19-28.

[42] J.A.C. Martins, M.M. Marques (Eds.), Contact Mechanics, Kluwer Academic Press, 2002.

[43] J.J. Moreau, Fonctionnelles sous-différentiables, CR Acad. Sci. Sér. I, Paris 257 (1963) 4117-4119.

[44] J.T. Oden, J.A.C. Martins, Models and computational methods for dynamic friction phenomena, Comp. Meth. Appl. Mech. Eng. 52 (1985) 527-534.

[45] A. Ould Khaoua, Modélisation théorique et numérique de problèmes de couches minces en élasticité, PhD thesis, Université Montpellier II, 1995.

[46] A. Ould Khaoua, F. Lebon, C. Licht, G. Michaille, Thin layers in elasticity: a theoretical and numerical study, in: Proceedings of the 1996 ESDA Conference, ASME, vol. 4, 1996, pp. 171-178.

[47] S. Pagano, P. Alart, F. Lebon, Un algorithme de décomposition convexe pour l'étude de structures en alliage à mémoire de forme. Application aux manchons de raccordement, Eur. J. Finite Elements 7 (1998) 365-400.

[48] P.D. Panagiotopoulos, J. Haslinger, The variational reciprocal approach to the Signorini problem with friction. Approximation results, Proc. Roy. Soc. Edinburgh 98A (1984) 365-383.

[49] F. Radjai, Multicontact dynamics, CR l'Ecole d'été Phys. Dry Granular Media, NATO ASI Ser. (1998) 305.

[50] M. Raous, Quasistatic Signorini problem with Coulomb friction and coupling to adhesion, in: New Developments in Contact Problems, Springer-Verlag, 1999, pp. 101-178.

[51] M. Raous, P. Chabrand, F. Lebon, Numerical methods for solving unilateral contact problem with friction, J. Theor. Appl. Mech. 7 (1988) 111-128.

[52] J. Sanchez-Hubert, E. Sanchez-Palencia, Introduction aux méthodes asymptotiques et à l'homogénéisation, Masson, 1992.

[53] P. Sonnenveld, P. Wesseling, P.M. de Zeeuw, Multigrid and conjugate gradient methods as convergence acceleration techniques, in: Multigrid Methods for Integral and Differential Equations, Clarendon Press, 1985, pp. 117-167.

[54] N. Stalin, K. Dang-Van, Numerical simulation of the sliding wear test in relation to material properties, Wear 203-204 (1997) 180-186.

[55] P. Suquet, Discontinuities and plasticity, in: Non-Smooth Mechanics and Applications, SpringerVerlag, 1988, pp. 279-340.

[56] J.J. Telega, Topics on unilateral contact problems of elasticity and inelasticity, in: Non-Smooth Mechanics and Applications, Springer-Verlag, 1988, pp. 341-462.

[57] J.J. Telega, Quasi-static Signorini's contact problem with friction and duality, Int. Ser. Num. Math. 101 (1991) 199-214.

[58] P. Wriggers, P. Panagiotopoulos (Eds.), New Developments in Contact Problems, Springer-Verlag, 1999.

[59] F. Zaittouni, Modélisation théorique et numérique d'interfaces, $\mathrm{PhD}$ thesis, Université Montpellier II, 2000.

[60] F. Zaittouni, F. Lebon, C. Licht, Etude théorique et numérique du comportement d'un assemblage de plaques, CR Acad. Sci. Sér. II, Paris, 2002. 